\title{
Neonatal Blood Carnitine Concentrations: Normative Data by Electrospray Tandem Mass Spectometry
}

\author{
DONALD H. CHACE, ROSER PONS, CLAUDIA A. CHIRIBOGA, DONALD J. MCMAHON, \\ INGRID TEIN, EDWIN W. NAYLOR, AND DARRYL C. DE VIVO
}

\begin{abstract}
Neo Gen Screening [D.H.C., E.W.N.], Division of BioAnalytical Chemistry and Mass Spectrometry, Bridgeville, PA 15017, U.S.A.; Departments of Neurology and Pediatrics [R.P., C.A.C., D.J.M., D.C.D.V.], Division of Child Neurology, Columbia University, New York, NY 10032, U.S.A.; and Department of Pediatrics [I.T.], Division of Neurology, The Hospital for Sick Children, The University of Toronto, Toronto, Ontario, Canada M5G1X8
\end{abstract}

\begin{abstract}
Despite a number of published reports, there is limited information about carnitine metabolism in the newborn. To establish normative data, we analyzed whole-blood carnitine concentrations in 24,644 newborns at age $1.85 \pm 0.95 \mathrm{~d}$ and umbilical cord whole blood and plasma carnitine concentrations in 50 full-term newborns. Total carnitine (TC), free carnitine (FC), and acylcarnitine (AC) were measured by electrospray tandem mass spectrometry. AC/FC ratios were derived from these measurements. The entire cohort was stratified according to TC values into a middle TC group representing $90 \%$ of the population and lower and upper TC groups representing 5\% of the population, respectively. Normative data were derived from the middle TC group of full-term infants $(N=19,595)$. TC was $72.42 \pm 20.75 \mu \mathrm{M}, \mathrm{FC}$ was $44.94 \pm 14.99 \mu \mathrm{M}$, AC was $27.48 \pm 8.05 \mu \mathrm{M}$, and $\mathrm{AC} / \mathrm{FC}$ ratio was $0.64 \pm 0.19( \pm \mathrm{SD})$. These values differed significantly from umbilical cord whole blood TC values of $31.27 \pm 10.54$ $\mu \mathrm{M}$ determined in 50 samples. No meaningful correlation was found between TC and gestational age or birth weight in any group. In controlled analyses, prematurity was not associated with TC levels, whereas low birth weight $(<2500 \mathrm{~g})$ and male
\end{abstract}

\section{ABSTRACT}

sex were significantly associated with higher TC levels. The association of low birth weight with higher TC values may be related to decreased tissue carnitine uptake. The sex effect may be related to hormonal influences on carnitine metabolism. Our study provides normative data of carnitine values measured by the highly precise method of electrospray tandem mass spectrometry in a large cohort of newborns and provides the basis for future studies of carnitine metabolism in health and disease states during the neonatal period. (Pediatr Res 53: 823-829, 2003)
Abbreviations
MS-MS, electrospray tandem mass spectrometry
TC, total carnitine
FC, free carnitine
AC, acylcarnitine
RBC, red blood cells
OR, Odds ratio
CI, $95 \%$ confidence interval
RE, radioenzyme

L-Carnitine serves as a carrier for acyl groups across the mitochondrial membrane. It is essential for the membrane transport and subsequent intramitochondrial $\beta$-oxidation of long-chain fatty acids and for the modulation of CoA homeostasis $(1,2)$. Adequate tissue levels of carnitine are crucial in the neonatal period, because mitochondrial oxidation of fatty

Received November 2, 2001; accepted November 29, 2002.

Correspondence: Darryl C. De Vivo, M.D., The Neurological Institute, 710 W 168th Street, New York, NY 10032, U.S.A.; e-mail: dcd1@columbia.edu

Supported in part by Sigma-Tau Pharmaceutical Inc. (Gaithersburg, MD), The Colleen Giblin Foundation for Pediatric Neurology Research, USPHS Grant RR 00645, and National Institutes of Health Grant RO1 DA11410.

DOI: 10.1203/01.PDR.0000059220.39578.3D acids is essential for the production of energy in newborns $(3-6)$.

L-Carnitine is derived mainly from exogenous sources and in part from endogenous synthesis (1). The main source of carnitine in the fetus seems to be transplacental transfer $(4,7,8)$ and in the newborn seems to be breast milk or carnitine-supplemented formulas (3, 9-11). Endogenous synthesis plays a minor role in early life because the activity of $\gamma$-butyrobetaine hydroxylase, the enzyme necessary for carnitine synthesis, is very low (12).

In tissues and body fluids, carnitine is present in free and esterified forms. The sum of free carnitine (FC) and acylcarnitine (AC) equals total carnitine (TC). The fraction of esterified carnitine varies depending on the tissue and on the metabolic status $(1,13,14)$. 
Reduction of FC and accumulation of $\mathrm{AC}$ is a common feature of inborn errors of metabolism affecting fatty acid oxidation and other mitochondrial disorders $(1,13,14)$. These diseases often present in the neonatal period and early infancy (1). Knowledge of carnitine metabolism in neonates is essential if these diseases are to be recognized and treated.

An extensive literature describes the need for carnitine supplementation in newborns $(9-11,15)$. Recommendations for carnitine supplementation, however, should be based on the knowledge of the normal carnitine values in this population and on the perinatal factors that can affect the concentration and the relative distribution of $\mathrm{FC}$ and $\mathrm{AC}$.

Only a few articles $(3,11,16-25)$ have reported carnitine concentrations in the perinatal period since the original report of Novack et al. (26). However the interpretation of the cumulative information from these reports is difficult because of several differences: 1) the study populations 2), technical methods 3), postnatal timing of samples, and 4) analyzed tissues (e.g. blood, plasma, serum). These differences have obscured the normal carnitine values, the evolution of carnitine values postnatally, and the definition of neonatal carnitine deficiency. In addition, the small number of subjects in each report limits the generalizability of the findings. In this report, we present the data collected from 24,644 consecutive newborns at age $1.85 \pm 0.95 \mathrm{~d}$. The data provide normal values of blood carnitine concentration in newborns and permit some speculation regarding perinatal factors that may influence carnitine homeostasis in this age group.

\section{METHODS}

The carnitine screening project was conducted in collaboration with Neo Gen Screening LLP (Pittsburgh, PA). This diagnostic service uses tandem mass spectrometry to screen for inborn errors of metabolism using newborn blood spots. Neo Gen Screening generated 25,592 consecutive carnitine screening results from newborn infants in the Pennsylvania region and their corresponding demographic and birth data. TC, FC, and $\mathrm{AC}$ were measured from filter paper blood spots using electrospray tandem mass spectrometry (MS-MS), as previously described $(27,28)$. The study lasted $16 \mathrm{mo}$. We were interested in obtaining normative data from a homogeneous cohort. In an attempt to eliminate any sample selection bias, only consecutive specimens were included.

From the original sample, 24,644 results were included for statistical analysis. A total of 948 blood specimens were discarded as unsatisfactory for demographic reasons; because of extraneous, nonconsecutive birth dates; or because of transfusion before newborn screening sampling. Demographic information in the sample included gestational age, birth weight, and time of sampling.

The statistical analyses were carried out using both SPSS (version 10.05 for Windows) and The SAS system for Windows Release 8.02 TS Level 02M0. Pearson Rho correlation coefficient and $t$ tests were used to assess continuous variables, and $\chi^{2}$ statistics were used for dichotomous or categorical variables. Strength of association was estimated by the odds ratio $(\mathrm{OR})$ and $95 \%$ confidence interval $(\mathrm{CI})$. Linear regression analyses (maximum square analyses) were carried out to identify covariates associated with TC.

We carried out two sets of analyses: one involved the entire cohort $(N=24,644)$, and the other involved only term infants $(N=21,686)$. To normalize the data further, we performed additional subgroup analyses by dividing the population into the upper $5 \%$, middle $90 \%$, and lower $5 \%$ according to TC values.

In addition, we studied 50 cord blood specimens from full-term infants delivered at Harlem Hospital Center in New York. Cord blood specimens were collected in a heparinized tube at the time of birth. The samples were gently mixed to obtain a uniform distribution of the cellular elements before an aliquot of whole blood was obtained for sampling. After this aliquot was obtained, the heparinized blood specimen was centrifuged to yield the plasma supernatant. All specimens were prepared and allowed to air-dry on filter paper on the day of retrieval (mean: 2-3 d after collection). These 50 samples were then analyzed by MS-MS, as above.

Fifty additional plasma samples were collected over a 1-mo period. These samples were submitted for carnitine analysis as indicated clinically. An aliquot of plasma was spotted on filter paper and air-dried for MS-MS assay. Another aliquot of plasma was assayed by the conventional radioenzyme (RE) assay (29). We compared plasma carnitine results provided by MS-MS to the measurements obtained by the RE technique (29).

These studies were approved by the Columbia University Institutional Review Board. The study subjects remained anonymous.

\section{RESULTS}

Descriptive analysis of the entire sample. A summary of descriptive statistics and the mean carnitine values for the entire sample of 24,644 newborns is depicted in Table 1 . The mean interval between birth date and the date of blood spot on filter card was $1.85 \pm 0.95 \mathrm{~d}$. The data were grouped into three populations on the basis of TC values: middle $90 \%$ (normal TC) population, upper 5\% (high TC) population, and lower 5\% (low TC) population. Carnitine values showed normal distribution in the middle $90 \%$ group with skewness and kurtosis of 0.56 and -0.43 , respectively.

In the normal TC population, girls and boys were equally represented (Table 1). A total of $83.6 \%$ of newborns were white, and $9.9 \%$ were black. The mean gestational age was $38.8 \pm 2.5 \mathrm{wk}$, and the mean birth weight was $3348 \pm 652 \mathrm{~g}$. There were no differences in mean birth weight or gestational age between the three groups of carnitine values (data not shown).

Univariate analyses of the entire sample. On stratified analyses, both low birth weight infants $(<2500 \mathrm{~g})$ and premature infants $(<37 \mathrm{wk}$ gestation) were significantly overrepresented in the high TC group compared with the normal TC (low birth weight $\mathrm{OR}=2.4,95 \% \mathrm{CI}=1.8-2.5 ; p<0.0001$ ) and premature infants $(\mathrm{OR}=1.5,95 \% \mathrm{CI}=1.3-1.8 ; p<$ 0.0001). 
Table 1. Summary of descriptive statistics for the total group of newborns and the full-term group of newborns

\begin{tabular}{|c|c|c|c|c|}
\hline & $\begin{array}{c}\text { Total group } \\
(N=24644)\end{array}$ & $\begin{array}{c}\text { High } 5 \% \\
(N=1232)\end{array}$ & $\begin{array}{c}\text { Middle } 90 \% \\
(N=22180)\end{array}$ & $\begin{array}{c}\text { Low } 5 \% \\
(N=1232)\end{array}$ \\
\hline $\mathrm{TC}^{*}$ & $74.63 \pm 28.97$ & $153.21 \pm 31.99$ & $72.54 \pm 20.82$ & $33.57 \pm 4.53$ \\
\hline $\mathrm{FC}^{*}$ & $46.85 \pm 21.19$ & $103.37 \pm 28.98$ & $45.24 \pm 15.16$ & $19.38 \pm 3.76$ \\
\hline $\mathrm{AC}^{*}$ & $27.77 \pm 10.01$ & $49.85 \pm 12.39$ & $27.30 \pm 8.05$ & $14.20 \pm 2.88$ \\
\hline $\mathrm{AC} / \mathrm{FC}$ & $0.64 \pm 0.19$ & $0.50 \pm 0.16$ & $0.64 \pm 0.19$ & $0.76 \pm 0.23$ \\
\hline Female & $48.6 \%$ & $39.4 \%$ & $48.4 \%$ & $60.9 \%$ \\
\hline Male & $51.4 \%$ & $60.6 \%$ & $51.6 \%$ & $39.1 \%$ \\
\hline \multicolumn{5}{|l|}{ Weeks gestation } \\
\hline $24-27$ & $0.2 \%$ & $0.1 \%$ & $0.2 \%$ & $0.6 \%$ \\
\hline $28-31$ & $1.0 \%$ & $1.7 \%$ & $0.9 \%$ & $2.4 \%$ \\
\hline $32-36$ & $8.1 \%$ & $11.3 \%$ & $7.9 \%$ & $8.2 \%$ \\
\hline $37-42$ & $88.0 \%$ & $83.9 \%$ & $88.3 \%$ & $85.8 \%$ \\
\hline$>42$ & $0.1 \%$ & $0.2 \%$ & $0.1 \%$ & $0.1 \%$ \\
\hline \multicolumn{5}{|l|}{ Birth weight (g) } \\
\hline $0-750$ & $0.2 \%$ & $0.0 \%$ & $0.2 \%$ & $0.2 \%$ \\
\hline $750-1500$ & $0.9 \%$ & $2.3 \%$ & $0.8 \%$ & $2.0 \%$ \\
\hline $1500-2500$ & $6.4 \%$ & $11.6 \%$ & $6.2 \%$ & $5.4 \%$ \\
\hline $2500-3500$ & $50.8 \%$ & $46.6 \%$ & $50.9 \%$ & $52.7 \%$ \\
\hline $3500-4500$ & $37.9 \%$ & $34.7 \%$ & $38.2 \%$ & $36.4 \%$ \\
\hline \multirow[t]{2}{*}{$>4500$} & $1.6 \%$ & $2.4 \%$ & $1.5 \%$ & $1.1 \%$ \\
\hline & $\begin{array}{c}\text { Full-term } \\
(N=21686)\end{array}$ & $\begin{array}{c}\text { High } 5 \% \\
(N=1034)\end{array}$ & $\begin{array}{c}\text { Middle } 90 \% \\
(N=19595)\end{array}$ & $\begin{array}{c}\text { Low } 5 \% \\
(N=1057)\end{array}$ \\
\hline $\mathrm{TC}$ & $74.36 \pm 28.58$ & $152.62 \pm 32.54$ & $72.42 \pm 20.75$ & $33.65 \pm 4.48$ \\
\hline $\mathrm{FC}$ & $46.41 \pm 20.73$ & $101.91 \pm 29.43$ & $44.94 \pm 14.99$ & $19.27 \pm 3.72$ \\
\hline $\mathrm{AC}$ & $27.95 \pm 10.00$ & $50.71 \pm 12.21$ & $27.48 \pm 8.05$ & $14.38 \pm 2.78$ \\
\hline $\mathrm{AC} / \mathrm{FC}$ & $0.65 \pm 0.19$ & $0.52 \pm 0.15$ & $0.64 \pm 0.19$ & $0.77 \pm 0.22$ \\
\hline
\end{tabular}

$* \mu \mathrm{mol} / \mathrm{L} \pm \mathrm{SD}$ in blood.

Statistically significant sex differences were noted in the TC groups (Table 1): girls were overrepresented in the low TC group $(60.9 \%$; $\mathrm{OR}=1.7,95 \% \mathrm{CI}=1.5-1.9 ; p<0.0001)$ and boys were overrepresented in the high TC group $(60.6 \%$; OR $=1.4,95 \% \mathrm{CI}=1.3-1.6 ; p<0.0001)$ when compared with the middle TC group.

Linear correlation analysis did not show meaningful correlation between TC and birth weight (g) or gestational age (weeks of gestation) in any of the groups analyzed. AC and
$\mathrm{AC} / \mathrm{FC}$ ratio, however, significantly correlated with birth weight in all groups and with gestational age only in the low TC group (Table 2).

Descriptive statistical analysis of the full-term group. To capture the representative normal term population of infants, we selected newborns $37-42 \mathrm{wk}$ of gestation from the original cohort $(N=21,686)$ and divided the group into three subgroups on the basis of TC levels (Table 1). Normative data were drawn from the middle $90 \%$ group of the full-term

Table 2. Correlation coefficient values between birth weight and blood carnitine concentrations in the total group of newborns and full-term newborns

\begin{tabular}{|c|c|c|c|c|c|c|}
\hline \multirow[b]{2}{*}{ Total group } & \multicolumn{2}{|c|}{ High $5 \%(N=1232)$} & \multicolumn{2}{|c|}{ Middle $90 \%(N=22180)$} & \multicolumn{2}{|c|}{ Low $5 \%(N=1232)$} \\
\hline & Rho & $p$ value & Rho & $p$ value & Rho & $p$ value \\
\hline \multicolumn{7}{|l|}{ Birth weight } \\
\hline $\mathrm{FC}$ & -0.095 & 0.0009 & -0.073 & 0.0001 & -0.121 & 0.0001 \\
\hline $\mathrm{AC}$ & 0.162 & 0.0001 & 0.088 & 0.0001 & 0.242 & 0.0001 \\
\hline $\mathrm{AC} / \mathrm{FC}$ & 0.215 & 0.0001 & 0.164 & 0.0001 & 0.237 & 0.0001 \\
\hline \multicolumn{7}{|l|}{ Gestational age } \\
\hline $\mathrm{FC}$ & -0.000 & 0.9995 & -0.001 & 0.8490 & -0.113 & 0.0001 \\
\hline $\mathrm{AC}$ & -0.001 & 0.7332 & 0.011 & 0.1087 & 0.215 & 0.0001 \\
\hline $\mathrm{AC} / \mathrm{FC}$ & -0.012 & 0.6892 & 0.013 & 0.0610 & 0.205 & 0.0001 \\
\hline Full-term & \multicolumn{2}{|c|}{$(N=1034)$} & \multicolumn{2}{|c|}{$(N=19595)$} & \multicolumn{2}{|c|}{$(N=1057)$} \\
\hline \multicolumn{7}{|l|}{ Birth weight } \\
\hline $\mathrm{TC}$ & 0.015 & 0.638 & -0.002 & 0.776 & 0.008 & 0.799 \\
\hline
\end{tabular}


population $(N=19,595)$ : TC values were $72.42 \pm 20.75 \mu \mathrm{M}$, FC values were $44.94 \pm 14.99 \mu \mathrm{M}, \mathrm{AC}$ values were $27.48 \pm$ $8.05 \mu \mathrm{M}$, and the $\mathrm{AC} / \mathrm{FC}$ ratio was $0.64 \pm 0.19$. The ratio of $\mathrm{AC} / \mathrm{FC}$, did not remain constant between the groups, and a significant difference was noted, with the ratio being higher in the low TC group $(0.77 \pm 0.22)$ and lower in the high TC group $(0.52 \pm 0.15 ; p<0.001)$ when compared with the middle TC group (Fig. 1).

Univariate analyses in the full-term group. In the full-term newborn group, girls were significantly overrepresented in the low TC group $(\mathrm{OR}=1.5,95 \% \mathrm{CI}=1.4-1.6 ; p<0.0001)$ and boys were overrepresented in the high TC group $(\mathrm{OR}=1.4$, $95 \% \mathrm{CI}=1.3-1.5 ; p<0.0001)$ when compared with the 90th percentile group. Low birth weight infants $(<2500 \mathrm{~g})$ were overrepresented in the high $\mathrm{TC}$ group $(\mathrm{OR}=2.1,95 \% \mathrm{CI}=$ $1.8-2.4 ; p<0.0001$; Table 2). Linear correlation analysis showed a modest positive correlation between $\mathrm{AC} / \mathrm{FC}$ ratio and birth weight in the normal and low TC groups (Table 2).

Multivariate analysis. In multivariate analysis of the entire cohort, low birth weight was significantly associated with higher TC values $(\beta=8.86, p=0.000)$ and male sex was significantly associated with higher TC values $(\beta=6.11 p=$ $0.000)$, whereas prematurity was not linked $(\beta=-1.04, p=$ 0.191; Table 3).

Cord blood analysis. The cohort of 50 full-term newborns for cord blood analysis was represented by residents of the Harlem community in North Manhattan. A total of $68 \%$ of newborns were black. The mean gestational age was $39.1 \pm$ $1.8 \mathrm{wk}$, and the average birth weight was $3336.0 \pm 518.8 \mathrm{~g}$. Table 4 summarizes the mean carnitine values for whole blood and plasma in this group of patients. Correlation analysis showed a positive correlation between whole blood and plasma TC levels $(\rho=0.365, p<0.01)$. Statistical analysis and comparison was not performed between whole-blood carnitine levels from this newborn cohort and the large cohort because of the differences in the two population sizes.

Comparison of methods (RE and MS-MS). The mean carnitine plasma values generated using both techniques are

Carnitine Distribution in Full Term Newborns

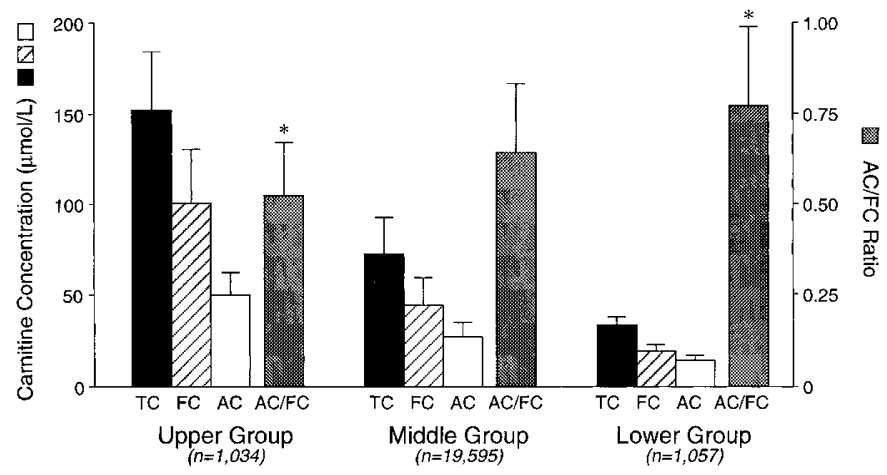

Figure 1. Blood carnitine distribution in full-term newborns. A comparison of blood carnitine profile in the full-term population. The middle group represents $90 \%$ of the population, and the upper and lower groups represent $5 \%$ of the population, respectively. The $\mathrm{AC} / \mathrm{FC}$ ratios in the upper and lower groups were significantly different $\left({ }^{*} p<0.001\right)$ when compared with the total group.
Table 3. Independent predictors of total blood carnitine in the entire cohort $(\mathrm{N}=24644)$

\begin{tabular}{lcccc}
\hline \multicolumn{1}{c}{ Variables } & $\beta$ Coefficient & Standard error & $p$ value & $\mathrm{R}^{2}$ \\
\hline Low birth weight & 8.856 & 0.895 & 0.000 & \\
Male & 6.110 & 0.374 & 0.000 & \\
Prematurity & -1.038 & 0.793 & 0.191 & \\
& & & & 0.016 \\
\hline
\end{tabular}

summarized in Table 5. Wilcoxon matched-pairs signed-rank test indicated a significant difference between TC and FC values measured by RE and MS-MS with $\mathrm{Z}=-3.725(p<$ $0.002)$ and $Z=-4.342(p<0.0001)$, respectively. An observable correlation was found in FC values measured by RE and MS-MS $(\rho=0.441, p<0.002)$.

A secondary analysis grouped specimens according to $\mathrm{FC}$ values generated by RE and MS-MS. Table 6 summarizes plasma carnitine values for each group on the basis of FC. For low values of $\mathrm{FC}(\leq 45.9 \mu \mathrm{M})$, the mean plasma carnitine values generated by RE and MS-MS were comparable. However, mean plasma carnitine values between the two methods deviated progressively for higher values of FC.

\section{DISCUSSION}

This study is based on the analysis of a homogeneous cohort, a large sample size, and the highly precise quantification method of MS-MS. This method has become the gold standard for newborn screening of amino acid, fatty acid, and organic acid disorders. Neo Gen Screening of Pittsburgh has applied MS-MS to routine newborn screening with a false-positive rate of only $0.26 \%$ (30). The aim of this study was to use this modern method to quantify carnitine concentrations in a large cohort of newborns.

To correlate the carnitine concentrations obtained with MS-MS to previously used methods, we measured carnitine in 50 plasma samples by MS-MS and by conventional RE. MS-MS values, although generally higher, correlate well with those measured by the RE method (Tables 5 and 6 ). The higher values observed by MS-MS suggest that this method measures carnitine over a larger range, especially for high values. The $\mathrm{RE}$ method is based on a standard curve from 25 to $75 \mu \mathrm{M}$ carnitine concentration. Higher values may be underestimated in this enzymatic assay.

Previously reported plasma TC values obtained within the first 2 wk of life (excluding values based on cord blood sampling) ranged between 13 and $37 \mu \mathrm{M}(11,18-21)$ versus a mean of $72.33 \mu \mathrm{M}$ in whole blood in our study. It is difficult to compare our results with previously reported results in newborns because the methods and samples differed. We sampled whole blood, whereas most other studies $(11,16,19-21,23$, 31) sampled plasma. This difference is a major confounding factor. On the basis of our studies in cord blood, whole-blood carnitine by MS-MS method is approximately two times higher than that measured in plasma (Table 4). Consequently, the previously reported neonatal plasma values seem to be comparable to our values in whole blood. This assumption is probably more accurate when the blood and plasma carnitine values are low for the reasons stated earlier. 
Table 4. Carnitine values ( $\mu \mathrm{mol} / \mathrm{L} \pm S D$ ) in whole blood and plasma from cord blood $(\mathrm{N}=50)$

\begin{tabular}{lllrr}
\hline & \multicolumn{1}{c}{ TC } & FC & AC & AC/FC \\
\hline Whole blood & $31.27 \pm 10.54$ & $21.59 \pm 10.52$ & $10.77 \pm 4.32$ & $0.52 \pm 0.16$ \\
Plasma & $15.86 \pm 5.60$ & $12.95 \pm 4.85$ & $2.91 \pm 1.18$ & $0.22 \pm 0.11$ \\
\hline
\end{tabular}

Table 5. Plasma carnitine values ( $\mu m o l / L)$ by RE and MS techniques

\begin{tabular}{lcccc}
\hline Technique & TC & FC & AC & AC/FC \\
\hline RE & $47.31 \pm 15.38$ & $36.65 \pm 15.78$ & $10.66 \pm 9.99$ & $0.44 \pm 0.55$ \\
MS/MS & $65.51 \pm 29.44$ & $52.21 \pm 23.61$ & $13.30 \pm 12.20$ & $0.29 \pm 0.24$ \\
\hline
\end{tabular}

Table 6. Plasma carnitine values ( $\mu$ mol/L) for specimens grouped according to FC values

\begin{tabular}{clll}
\hline Groups based on FC & \multicolumn{1}{c}{ TC } & \multicolumn{1}{c}{ FC } \\
\hline Low values set $(N=21) \mathrm{FC} \leq 45.9$ & RE: $43.38 \pm 13.98$ & RE: $29.39 \pm 14.99$ & RE: $13.99 \pm 11.16$ \\
& MS: $39.78 \pm 8.83$ & MS: $29.80 \pm 7.63$ & MS: $9.98 \pm 6.54$ \\
Intermediate values set $(N=19) \mathrm{FC} \geq 46.0, \mathrm{FC} \leq 75.9$ & RE: $49.67 \pm 17.81$ & RE: $40.51 \pm 14.55$ & RE: $9.16 \pm 9.52$ \\
& MS: $71.02 \pm 16.54$ & MS: $58.69 \pm 9.22$ & MS: $12.34 \pm 11.01$ \\
High values set $(N=9) \mathrm{FC} \geq 76.0$ & RE: $52.66 \pm 11.48$ & RE: $46.91 \pm 12.11$ & RE: $5.74 \pm 5.87$ \\
& MS: $106.35 \pm 15.20$ & MS: $88.19 \pm 11.58$ & MS: $18.17 \pm 15.10$ \\
\hline
\end{tabular}

Recently, whole-blood carnitine values measured by MS-MS in newborns were reported by two other groups of investigators $(24,25,32)$. Meyburg et al. (24) studied 70 full-term newborns in the fifth day of life. TC and FC values were lower than ours $(55.7 \pm 1.6 \mu \mathrm{M}$ and $30.6 \pm 10.6 \mu \mathrm{M}$, respectively), whereas AC was comparable to our values (25.1 $\pm 6.4 \mu \mathrm{M}$; Table 1). Several factors could account for these differences. First, the number of newborns studied was much smaller than in our study and thus more prone to sample size biases. Second, several studies have shown changes in TC, FC, and $\mathrm{AC}$ within the first days of life $(3,9,11,16,19,21,24,26$, 33). Therefore, timing of blood sampling (day of life 1.85 versus day of life 5) might explain some differences in the reported carnitine concentrations. Third, methodological factors, such as differences in adjusting for blood volume, in extraction efficiency or in hydrolysis may also play a role (27). Contamination of acetylcarnitine (C2) peak by glutamic acid also can falsely increase the value of $\mathrm{AC}$ (Chace $\mathrm{DH}$, unpublished data).

The second group of investigators $(25,32)$ found that the median value of $\mathrm{FC}$ from whole capillary blood of newborns at age $2 \mathrm{~d}$ was $26.6 \mu \mathrm{M}(N=13,311)$. Their lower FC values, once again, could be explained by the previously mentioned methodological differences and by the integrity of the internal standards.

In terms of the $\mathrm{AC} / \mathrm{FC}$ ratio, our values are comparable to those in previous studies. The $\mathrm{AC} / \mathrm{FC}$ ratio in neonates in these studies ranges from 0.31 to $1.2(19-21,24)$, and in our study this ratio was $0.64 \pm 0.19$. A constant finding among neonatal carnitine studies is the higher proportion of AC in the TC pool, compared with later in life. This ratio reflects the intramitochondrial relationship between acyl-CoA and free CoA and is very sensitive to metabolic changes in the mitochondria (13). The normal plasma $\mathrm{AC} / \mathrm{FC}$ ratio is 0.25 (13). Although this reference value does not take into consideration fluctuations related to feeding or fasting states, it is considered abnormal when the ratio is $>0.4$, indicating accumulation of $\mathrm{AC}$ and/or reduced FC. This ratio is often abnormal in inborn errors of metabolism such as fatty acid oxidation deficiencies, other mitochondrial disorders, and organic acidurias $(1,13,14)$. The high $\mathrm{AC} / \mathrm{FC}$ ratio noted in neonates is thought to reflect the increased production of acyl-CoA derivatives, mainly acetyl$\mathrm{CoA}$, produced by the normally enhanced fatty acid oxidation in the newborn period $(3,5,6,19,26)$ when the neonate is first exposed to fasting.

After dividing our cohort into high, low, and middle TC groups (Table 1), we noted that the $\mathrm{AC} / \mathrm{FC}$ ratio was significantly higher in the group of newborns with low TC (Fig. 1). This finding indicates that this group of newborns has a higher proportion of acyl-CoA species reflecting differences in intermediary metabolism. These differences may be due to acquired conditions or to inborn errors of metabolism. Inborn errors of metabolism associated with changes in carnitine homeostasis may present during infancy or in the early neonatal period. Recently, two patients with carnitine transporter defect were found to have very low carnitine concentrations when blood spots from the neonatal period were analyzed retrospectively (25). Also, recently, at our institution, a 3-d-old girl presented with weak cry, decreased movements, hypotonia, and sleepiness associated with hypoglycemia, increased lactic acid, hyperammonemia, and elevated muscle and liver enzymes. Her carnitine levels on neonatal screening showed TC of $52 \mu \mathrm{M}$, $\mathrm{FC}$ of $4 \mu \mathrm{M}$, and $\mathrm{AC}$ of $48 \mu \mathrm{M}$. Later, biochemical analysis of cultured fibroblasts showed deficiency of carnitine/AC translocase activity. Some of these disorders are now diagnosed by neonatal screening. Interestingly, two newborns with mediumchain acyl-CoA dehydrogenase deficiency and one with isovaleric acidemia were detected in our cohort, but none of them showed abnormalities in blood carnitine levels (data not shown). This variability in the carnitine levels of patients with inborn errors of metabolism in the neonatal period indicates that multiple factors influence carnitine homeostasis during this developmental stage. Follow-up studies of infants with low neonatal carnitine concentrations and/or high $\mathrm{AC} / \mathrm{FC}$ ratios are necessary and are planned to identify possible acquired or genetic conditions associated with low neonatal TC levels and high proportions of acylated carnitine. 
In our analysis of whole blood and plasma carnitine levels from cord blood, the plasma values obtained (Table 4) were comparable to those reported in the literature $(17,19,20,22$, $23,34)$, probably because the timing of sampling was identical for all of the studies. All of the carnitine fractions and the proportion of acylated carnitine were higher in whole blood than in plasma (22) Table 4). This finding indicates that there is a concentration of carnitine and, especially, $\mathrm{AC}$ in the cellular compartment, consistent with previous studies showing higher AC concentrations in red blood cells (RBC) compared with plasma $(22,32,35)$. The role of carnitine in $R B C$ is unclear because fatty acid oxidation is not used as a source of energy. It is thought that carnitine concentrations in RBC are already fixed during hematopoiesis in reticulocytes $(22,35)$. At this stage, carnitine is necessary for fatty acid transport, whereas in mature erythrocytes, it is thought to be important for membrane stability and several membrane functions (22). It has been suggested that $\mathrm{AC}$ in blood cells might act as a reservoir of immediately accessible activated acyl groups (36).

Our analysis showed that whole-blood TC, FC, and AC levels in cord blood were lower $(43 \%, 48 \%$, and $39 \%$, respectively) than the levels in capillary blood on day of life 1.85. A comparison between these values has to take into account the large difference in the populations studied and the sample size. Nevertheless, these results are suggestive of an increase in blood carnitine concentrations during the first $2 \mathrm{~d}$ of life. Although a recent study found an increase only in $\mathrm{AC}$ at day of life 5 (24) and another one found no differences at day of life 4 (37), a similar increase in blood carnitine concentration as in our study has been reported previously $(3,19)$. The increase in carnitine values soon after birth may be related to the physiologic metabolic changes occurring in the early neonatal period, such as the dramatic induction of fatty acid oxidation, hepatic ketogenesis, and gluconeogenesis. These metabolic changes occur in response to birth stress (cold exposure, catecholamine secretion, hypoxia, and cord cutting), to extrauterine metabolic demands, and to the change in diet (high-fat/low-carbohydrate diet with $>90 \%$ of milk fat in the form of triglycerides containing mainly long-chain fatty acids) $(5,38-44)$. The increase in carnitine necessary to support these metabolic demands may be derived from carnitine intake $(3,11,19)$ or tissue release (45).

In addition to providing normative data, our large cohort has enabled us to perform various statistical analyses to examine the association of carnitine values with sex, gestational age, and birth weight.

Carnitine and sex. TC levels were lower in girls and higher in boys (Table 3). These findings are consistent with other reports and suggest hormonal influences in the carnitine status. Hormonal regulation of serum carnitine levels has been previously demonstrated in rats (46). In humans, a significant negative correlation between carnitine concentration and estradiol was found in girls, whereas no correlation with testosterone was found in boys (47). Adult carnitine levels are reached at an older age in male compared with female adults (47) with higher concentrations of carnitine in plasma, muscle, and urine in male adults $(46,48)$.
Carnitine and gestational age. There was no correlation between TC and gestational age (Table 2). Univariate analysis showed that premature infants were overrepresented in the high TC group, but controlled analysis failed to show a significant association between prematurity and blood TC (Table 3). There is no agreement in the literature regarding the concentration of fetal blood carnitine with advancing gestation. Some reports show a significant decrease of fetal blood carnitine levels in the later stages of gestation $(16,17,20,21,23)$, whereas others have shown no changes in fetal blood carnitine levels during gestation $(24,26)$. These observations have been made in newborns delivered prematurely and may not be an accurate representation of the blood carnitine concentration at that respective age of gestation under healthy conditions.

Carnitine and birth weight. It has been reported that skeletal muscle carnitine concentration increases significantly with birth weight (49). Regarding blood carnitine, however, some authors reported no significant correlation with birth weight $(17,18)$, whereas others found a significant positive correlation between carnitine levels and birth weight in premature infants $<950-1800 \mathrm{~g}(16,20)$. More recently, Giannacopouolou et al. (23) found a negative correlation between plasma carnitine and birth weight and Meyburg et al. (24) found a positive correlation between whole-blood carnitine fractions and birth weight. In our study, there was no meaningful correlation between TC and the continuous variable of birth weight. The $\mathrm{AC} / \mathrm{FC}$ ratio showed a modest positive correlation, suggesting a higher proportion of esterified carnitine with increased birth weight (Table 2). On controlled analysis, low birth weight was significantly associated with higher TC values (Table 3 ).

We speculate that the observed association between low birth weight and high blood TC may be due to reduced tissue mass and/or immature carnitine transporter in low birth weight infants, leading to lower tissue carnitine and higher blood carnitine levels. Consistent with this speculation, Penn et al. (50) found less tissue carnitine in low birth weight compared with normal weight newborns with the same gestational age.

\section{CONCLUSION}

In summary, we present normative data for whole-blood carnitine values in neonates. These data can be used for the assessment of carnitine status and the diagnosis of carnitine deficiency in the neonatal period. Future investigations need $l$ ) to establish a correlation between whole-blood carnitine values and tissue carnitine stores, 2) to evaluate the serial changes of whole-blood carnitine after birth, 3) to establish the predictive value of extreme neonatal blood values of carnitine, and 4) to analyze blood carnitine changes as a surrogate marker for immaturity and/or perinatal stress.

Acknowledgments. We thank Alice Kwan for data management and Drs. Piero Rinaldo, Salvatore DiMauro, and Leonidas Stefanis for critical comments during the conduct of these studies.

\section{REFERENCES}

1. Pons R, De Vivo DC 1995 Primary and secondary carnitine deficiency syndromes. J Child Neurol 10(suppl):2S8-2S24 
2. Bremer J 1983 Carnitine: metabolism and functions. Physiol Rev 63:1420-1449

3. Warshaw JB, Curry E 1980 Comparison of serum carnitine and ketone body concentration in breast- and in formula-fed newborn infants. J Pediatr 97:122-125

4. Schimdt-Sommerfeld E, Penn D, Sodha RJ, Progler M, Novak M, Schneider H 1985 Transfer and metabolism of carnitine esters in the in vitro perfused human placenta. Pediatr Res 19:700-706

5. Girard J, Ferre P, Pegorier JP, Duee PH 1992 Adaptations of glucose and fatty acid metabolism during perinatal period and suckling-weaning transition. Physiol Rev 72:507-562

6. Arenas J, Rubio JC, Martin MA, Campos Y 1998 Biological roles of L-carnitine in perinatal metabolism. Early Hum Dev 53(suppl)S43-S50

7. Novack M, Monkus EF, Chung D, Buch M 1981 Carnitine in the perinatal metabolism of lipids. I. Relationship between maternal and fetal plasma levels of carnitine and acylcarnitines. Pediatrics 67:95-100

8. Schimdt-Sommerfeld E, Penn D, Wolf H 1981 The influence of maternal fat metabolism on fetal carnitine levels. Early Hum Dev 5:233-242

9. Borum PR 1995 Carnitine in neonatal nutrition. J Child Neurol 10(suppl):2S25-2S31

10. Rebouche CJ 1992 Carnitine function and requirements during the life cycle. FASEB J 6:3379-3386

11. Campoy C, Bayes R, Peinado JM, Rivero M, Lopez C, Molina-Font JA 1998 Evaluation of carnitine nutritional status in full-term newborn infants. Early Hum Dev 53(suppl)S149-S164

12. Rebouche CJ, Engel AG 1980 Significance of renal gamma-butyrobetaine hydroxylase for carnitine biosynthesis in man. J Biol Chem 255:8700-8705

13. Bohles HJ, Evangeliou A, Bervoets K Eckert S, Sewell A 1994 Carnitine esters in metabolic disease. Eur J Pediatr 153(suppl 1):S57-S61

14. Sewell AC, Bohles HJ 1995 Acylcarnitines in intermediary metabolism. Eur J Pediat 154:871-877

15. Borum PR 1985 Role of carnitine during development. Can J Physiol Pharmacol 63:571-576

16. Battistella PA, Vergani L, Donzelli F, Rubaltelli F, Angelini C 1980 Plasma and urine carnitine levels during development. Pediatr Res 14:1379-1381

17. Shenai JP, Borum PR, Mohan P, Donlevy C 1983 Carnitine status at birth of newborn infants of varying gestation. Pediatr Res 17:579-582

18. Cederblad G, Niklasson A, Rydgren B, Altbertsson-Wikland K, Olegard R 1985 Carnitine in maternal and neonatal plasma. Acta Pediatr Scand 74:500-504

19. Schimdt-Sommerfeld E, Werner D, Penn D 1988 Carnitine plasma concentrations in 353 metabolically healthy children. Eur J Pediatr 147:356-360

20. Sachan DS, Smith RB, Plattsmier J, Lorch V 1989 Maternal cord, and neonata carnitine correlations and lipid profiles of various birth weight infants. Am J Perinato $6: 14-17$

21. Milana A, Mazzone D 1995 Aspetti peculiari della carnitina nel periodo neonatale. Clin Ter 146:21-29

22. Schoderbeck M, Auer B, Legenstein E, Genger H, Sevelda P, Salzer H, Marz R, Lohninger A 1995 Pregnancy-related changes of carnitine and acylcarnitine concentration of plasma and erythrocytes. J Perinat Med 23:477-485

23. Giannacopoulou Ch, Evangeliou A, Matalliotakis I, Relakis K, Sbirakis N, Hatzidaki E, Koumandakis E 1998 Effects of gestation age and of birth weight in the concentration of carnitine in umbilical plasma. Clin Exp Obstet Gynecol 25:42-45

24. Meyburg J, Shulze A, Kohlmueller D, Linderkamo O, Mayatepek E 2001 Postnata changes in neonatal acylcarnitine profile. Pediatr Res 49:125-129

25. Wilcken B, Wiley V, Sim KG, Carpenter K 2001 Carnitine transporter defect diagnosed by newborn screening with electrospray tandem mass spectrometry. J Pediatr 138:581-584

26. Novack M, Wieser PB, Buch M, Hahn P 1979 Acetylcarnitine and free carnitine in body fluids before and after birth. Pediatr Res 13:10-15

27. Chace DH, Hillman SL, Van Hove JLK, Naylor E 1997 Rapid diagnosis of MCAD deficiency: quantitative analysis of octanoylcarnitine and other acylcarnitines in newborn blood spots by tandem mass spectrometry. Clin Chem 43:2106-2113
28. Chace DH, DiPerna JC, Mitchell Brenda L, Sgrol B, Hofman LF, Naylor EW 2001 Electrospray tandem mass spectrometry for analysis of acylcarnitines in dried postmortem blood specimens collected at autopsy from infants with unexplained cause of death. Clin Chem 47:1166-1182

29. McGarry JD, Foster DW 1976 An improved and simplified radioisotopic assay for the determination of free and esterified carnitine. J Lipid Res 17:277-281

30. Levy HL 1998 Newborn screening by tandem mass spectrometry: a new era [editorial] Clin Chem 44:2401-2402

31. Bernardini I, Evans MI, Nicolaides KH, Economides DL, Gahl WA 1991 The fetal concentrating index as a gestational age-independent measure of placental dysfunction in intrauterine growth retardation. Am J Obstet Gynecol 164:1481-1490

32. Wiley V, Carpenter K, Wilcken B 1999 Newborn screening with tandem mass spectrometry: 12 months experience in NSW Australia. Acta Paediatr Suppl 432:48-51

33. Deufel T 1990 Determination of L-carnitine in biological fluids and tissues. J Clin Chem Clin Biochem 28:307-311

34. Akisu M, Bekler C, Yalaz M, Huseyinov A, Kultursay N 2001 Free carnitine concentrations in cord blood in preterm and full-term infants with intrauterine growth retardation. Pediatr Int 43:107-108

35. Cooper MB, Forte CA, Jones DA 1988 Carnitine and acetylcarnitine in red blood cells. Biochim Biophys Acta 959:100-105

36. Cress AP, Fraker PJ, Bieber LL 1989 Carnitine and acylcarnitine levels of human peripheral blood lymphocytes and mononuclear phagocytes. Biochim Biophys Acta 992:135-139

37. Hromadova M, Parrak V, Huttova M, Danova K, Urvolgyova M 1994 Carnitine level and several lipid parameters in venous blood of newborns, cord blood and maternal blood and milk. Endocr Regul 28:47-52

38. Augenfeld J, Fritz IB 1970 Carnitine palmitoyltransferase activity and fatty acid oxidation by livers from fetal and neonatal rats. Can J Biochem 48:288-294

39. Warshaw JB 1970 Cellular energy metabolism during fetal development. III Deficient acetyl-CoA synthetase, acetylcarnitine transferase and oxidation of acetate in the fetal bovine heart. Biochim Biophys Acta 223:409-415

40. Ballard FJ 1971 Gluconeogenesis and the regulation of blood glucose in the neonate In: Rodriguez RR, Valance-Owen J (eds) Diabetes. Excerpta Medica, Amsterdam, pp $592-600$

41. Hahn P, Skala JP 1975 The role of carnitine in brown adipose tissue of suckling rats. Comp Biochem Physiol B 51:507-515

42. Dils RR, Parker DS 1982 Metabolic aspects of lactation and the supply of nutrients to the young In: Jones CT (ed) Biochemical Development of the Fetus and Neonate. Elsevier, Amsterdam, pp 573-590

43. Girard J 1990 Metabolic adaptations to change of nutrition at birth. Biol Neonate 58(suppl 1):3-15

44. De Vivo DC, Tein I, Reichmann H 1991 Idiosyncratic reactions to valproate: clinical risk patterns and mechanisms of toxicity. In: Levy RH, Penry JK (eds) Age Related Changes in Fatty Acid Oxidation. Raven Press, New York, pp 13-18

45. French TJ, Goode AW, Sugden MC 1986 Blood carnitine concentrations are increased after surgical stress. Biochim Biophys Acta 882:133-135

46. Borum PR 1978 Variation in tissue carnitine concentrations with age and sex in the rat. Biochem J 176:677-681

47. Takiyama N, Matsumoto K 1998 Age- and sex-related differences of serum carnitine in a Japanese population. J Am Coll Nutr 17:71-74

48. Cederblad G 1976 Plasma carnitine and body composition. Clin Chim Acta 67:207212

49. Shenai YP, Borum PR 1984 Tissue carnitine reserves of newborn infants. Pediatr Res 18:670-681

50. Penn D, Ludwigs B, Schmidt-Sommerfeld E, Pascu F 1985 Effect of nutrition on tissue carnitine concentrations in infants of different gestational ages. Biol Neonate 47:130-135 